\title{
An Organized Effort is Needed to Fast Track the Development of Cancer Biomarkers
}

\author{
Sam Hanash
}

Published online: 26 February 2008

(C) Humana Press 2008

A vast amount of "omic" data has been accumulated for most cancer types that are potentially relevant to the development of protein-based cancer biomarkers. Most such data are derived from transcriptomic or proteomic analysis of tumor tissue, tumor cell lines, or biological fluids either proximal to tumors such as effusions or distal, notably serum, and plasma. Protein biomarker candidates embedded in these data may be relevant to the diagnosis of cancer through blood-based testing or may have utility to classify tumors, predict response to therapy, or help monitor disease progression, regression, and recurrence. The question is how do we fast-track biomarker development given the availability of so much pertinent data?

While there are no simple answers, two important resources would help fast-track the process substantially. One is the availability of specimen resources and the other is the availability of affinity capture agents. These resources are particularly needed for proteomic studies aimed at developing blood-based cancer biomarkers but are also needed for biomarker development across the board. Highquality standardized reference specimens meticulously procured and processed under exacting conditions to preserve their contents, and therefore their value, are needed to rigorously test or triage candidate markers. Affinity capture agents, notably antibodies, are also needed to allow investigations of candidate markers in ways that are not readily feasible with the current standard tool for proteomics, namely mass spectrometry. Antibodies allow elucidation of tissue distribution and subcellular localization of the proteins they targeted and assessment of their

S. Hanash $(\bowtie)$

Fred Hutchinson Cancer Research Center,

Seattle, WA, USA

e-mail: shanash@fhcrc.org occurrence as part of complexes and allow development of high throughput assays for their measurement using various assay formats. The availability of these two important resources for biomarker development would allow testing of candidate biomarkers using standardized tissue specimen and standardized reagents. As a result, meaningful performance comparisons can be made between biomarker candidates derived from heterogeneous studies to aid in developing marker panels, comparing performance of biomarkers between disease groups and their performance for specific applications from early diagnosis to disease classification and monitoring.

The next question is how to facilitate the development of these two important resources for fast-tracking biomarkers? An organized effort is necessary for this purpose that may benefit from designating funds specifically to this effect, both for specimen and antibody procurement and for distribution to biomarker developers. Funding sources for cancer research consist primarily of the National Institutes of Health, notably the National Cancer Institute, Industry and a multitude of philanthropic organizations and individual contributors committed to the fight against cancer in general or specific organ types of cancer in particular. A cooperative effort among the stakeholders to provide funding for these two resources would be highly beneficial. A case in point is the Biomarkers Consortium (http://www. biomarkersconsortium.org) which is a public-private partnership managed by the Foundation for the National Institutes of Health that aims to facilitate biomarker development. Important objectives of the Consortium include facilitating drug development through the application of biomarkers and helping achieve personalized medicine through predictive markers. While the Consortium goals do not particularly emphasize the development of specimen resources and affinity capture agents, the 
formation of this public-private consortium supports the view that stakeholders can work together to fast-track biomarker development, and therefore, initiatives to develop these two important resources would garner substantial support.

While organizations, notably the National Cancer Institute, have recognized the need for specimen resources and affinity capture agents, plans for developing these two resources remain sketchy. Given the relevance of these resources to biomarker development, and more broadly to translational research, concrete steps to be taken for specimen collection could include the following:

1. Clinical trials should be expected to include specimen collections for the purpose of biomarker development and/or testing, with adequate support allocated to specimen collection.

2. Programs with a significant clinical/translational research component should be expected to develop specimen resources as part of their activities through a set aside or other equally effective mechanism, specifically for funding these activities as opposed to vague expectations that these needs would be met. A good example is the NCI Early Detection Research Network which has developed a process and allocated funds for building and sharing a specimen resource for early detection applications of biomarkers and for developing a related informatics infrastructure (http://www.edrn. nci.nih.gov).

3. A mechanism to deposit/share specimen resources with a credit system to this effect should be developed.

While these ideas may not be novel and there are certainly scenarios in which they are currently being applied as illustrated, the emphasis here is on availability of specimens specifically for biomarker development that are collected under standardized operating procedures with the intent to distribute specimens to qualified investigators and projects.

The development of affinity capture agents for broad applications including biomarker research has certainly been considered and has been found to be quite challenging. The scope of such an effort may be difficult to define. Potential technologies are quite varied with throughput, cost and reach-through representing important issues. Yet, some creative solutions are beginning to emerge. The effort could be divided along tumor types or pathways for which biomarkers are sought. Stakeholders would then take on the task of assembling promising candidates for which affinity capture agents are needed from available data. Until an effective or a dominant alternative strategy for making capture agents emerges, polyclonal and monoclonal antibodies directed at several epitopes per candidate biomarker would be the preferred choice. A case in point is the development of antibodies directed at pancreatic cancer candidate markers through support from the Lustgarten foundations (http://www.lustgarten.org). If the numerous foundations committed to the fight against cancer adopt the development of antibodies against candidate biomarkers for the cancer type they are committed to fight, the problem of availability of antibodies against candidate cancer markers would be effectively addressed. The vast collection of resulting antibodies would be of substantial benefit for all aspects of research.

There remains numerous issues pertaining to execution, which would also need to be addressed. Not to be overlooked are the responsibilities of investigators that request to use such resources. In return for access to these resources, there has to be a commitment to share data and findings regarding marker candidate and antibody performance. It is through such organized efforts that cancer biomarker development and in particular, the promise of proteomics, can be fulfilled. 